\title{
Receiver Mobility in Vehicular Named Data Networking
}

\author{
Joao M. Duarte \\ University of Bern, Switzerland \\ IC, University of Campinas, Brazil \\ duarte@inf.unibe.ch
}

\author{
Torsten Braun \\ University of Bern, Switzerland \\ braun@inf.unibe.ch
}

\author{
Leandro A. Villas \\ IC, University of Campinas, Brazil \\ leandro@ic.unicamp.br
}

\begin{abstract}
This work investigates the topic of Vehicular Named Data Networking (VNDN). We propose a new VNDN routing protocol and address the negative effects caused by receiver mobility. In particular, we identify the problem of Reverse Path Partitioning (RPP) that often prevents Data messages from reaching Content Requesters, degrading application performance. To mitigate RPP we propose a mechanism called Auxiliary Forwarding Set (AFS). AFS takes several mobility factors as inputs and extends the NDN core philosophy by identifying an extra set of eligible nodes to forward Data messages whenever retransmissions are required due to RPP. Simulation results show that AFS is an efficient and scalable solution to improve VNDN application performance regardless of receiver mobility.
\end{abstract}

\section{CCS CONCEPTS}

- Networks $\rightarrow$ Routing protocols; Network simulations; Network mobility; In-network processing;

\section{KEYWORDS}

Vehicular Ad-hoc Networks, Named-Data Networking, Vehicular Named-Data Networking, Receiver mobility

ACM Reference format:

Joao M. Duarte, Torsten Braun, and Leandro A. Villas. 2017. Receiver Mobility in Vehicular Named Data Networking. In Proceedings of ACM SIGCOMM 2017 Workshop on Mobility in the Evolving Internet Architecture, UCLA, Los Angeles USA, August 2017 (MobiArch'2017), 6 pages.

DOI: $10.475 / 123 \_4$

\section{INTRODUCTION}

The Interest/Data messages model applied in Named Data Networking (NDN) [1], as well as in other Information Centric Networking (ICN) architectures, provides a suitable communication approach for Vehicular Ad-Hoc Network (VANET) applications, especially when contents are of interest to only a portion of the vehicles in a certain region.

NDN allows content to be produced and delivered on-demand, thus, supporting the deployment of a new set of VANET applications such as real time video streaming as well as gaming on the go. These new kinds of applications present higher performance requirements when compared to more traditional VANET applications such as

Permission to make digital or hard copies of part or all of this work for personal or classroom use is granted without fee provided that copies are not made or distributed for profit or commercial advantage and that copies bear this notice and the full citation on the first page. Copyrights for third-party components of this work must be honored. For all other uses, contact the owner/author(s).

MobiArch'2017, UCLA, Los Angeles USA

(C) 2016 Copyright held by the owner/author(s). 123-4567-24-567/08/06 .. \$15.00 DOI: $10.475 / 123 \_4$ accident and traffic condition warnings, which have been efficiently investigated in other works, including [2].

Compared to IP networks, NDN provides better support to VANET applications, since it does not have to deal with IP network requirements including domain name services, network masks, and default gateways as well as maintaining neighbor lists. This is of particular importance as VANET characteristics such as highly dynamic topologies and frequent disconnections, impose excessive overhead to maintain up to date routing tables. Besides, the NDN in-network caching property allows nodes to retrieve content from the closest available provider (i.e. either the original producer or a neighbor node having a cached copy of the content), thus decreasing content delivery delay [3].

Despite the NDN advantages, mobility factors impose several challenges to the deployment of NDN over VANETs in terms of application performance and resource management. In our previous work [4] we described various VANET mobility possibilities, from scenarios without mobility to receiver or/and source mobility.

In this work we adapt NDN to vehicular networks, we develop a Vehicular Named Data Networking (VNDN) routing protocol to route Interest and Data messages, we investigate how receiver mobility affects VNDN application performance, and we propose a solution to address a problem that we have identified and named as Reverse Path Partitioning (RPP). RPP frequently prevents Data messages from reaching Content Requesters, compromising application performance and is caused by variations of vehicle speeds, variable inter-vehicle transmission ranges, and signal attenuation due to the conditions of the wireless communication medium.

Our solution has the following objectives: (i) Provide high Interest Satisfaction Rates (ISR) (i.e. content received in response to Interest messages sent) by addressing RPP; (ii) Keep low average content delivery delay; (iii) Efficiently manage load in the wireless communication channel by limiting the number of vehicles required to forward Interest and Data messages.

The remainder of this paper describes the main contributions of related works in Section 2, our NDN adaptation to VANETs in Section 3, and the proposed VNDN routing protocol in Section 4. The RPP problem as well as AFS, the proposed solution to address the RPP effects, are described in Section 5. Section 6 presents the performance evaluations and Section 7 concludes this work.

\section{RELATED WORK}

The work in [5] presents a Content Centric Networking (CCN) framework for vehicular applications, which is compliant with the Wireless Access in Vehicular Environments (WAVE) architecture. It addresses the broadcast storm and message redundancy problems and Data messages include a provider information field to identify the best Content Providers. The FIB table is suppressed and Interest/Data messages are exchanged through broadcast. 
In [6] the authors modify some of the NDN functions to accommodate to VANETs. All messages are broadcast and to facilitate fast data dissemination, vehicles cache received content regardless of whether it was requested or not. Similarly to [5], the broadcast storm and message redundancy problems are addressed and the FIB table is suppressed since building and maintaining FIB in VANETS incurs high communication costs.

The works described above present substantial contributions for the deployment of ICN over VANETs. The broadcast storm and message redundancy problems are addressed. However, unlike in our work, the effects of receiver mobility, which are crucial for the performance of ICN over VANETs, are not considered in these works.

The work in [7] proposes a hop counter scheme to decrease the number of Data messages forwarded while achieving similar ISR compared to plain VNDN. This work does not focus on providing high ISRs in scenarios with high mobility, which is our main goal.

\section{VEHICULAR NAMED DATA NETWORKING}

In this section we describe how we adapt NDN to VANETs. NDN [1] presents two types of messages. (i) Interest messages, to request content and (ii) Data messages, to deliver content. In addition, NDN maintains three types of data structures. (i) Content Store (CS), for caching incoming content; (ii) Pending Interest Table (PIT), to keep track of forwarded Interest messages and (iii) Forwarding Information Base (FIB), to store outgoing interfaces to forward Interest messages.

NDN was originally designed for static scenarios. Therefore, its deployment over VANETs with high mobility requires some modifications in order to provide services with high performance. However, to preserve the NDN core philosophy we aim to perform as few modifications as required. Compared to [6], in our case, vehicles only store requested content, since not all vehicles are interested in all content, and Interest messages are routed towards Content Providers, instead of being broadcast in all directions.

In our VNDN approach, a vehicle can play three main roles: (i) Content Requester, when it requests a content object; (ii) Interest or Data Forwarder, when it forwards either an Interest or a Data message; and (iii) Content Provider, when the vehicle provides the requested content object.

In VANETs, maintaining up to date FIB entries connecting Content Requesters to Content Providers may be too costly, due to frequent changes in connectivity. With these in mind, similarly to [5] and [6] the FIB is not used.

Our approach assumes that all vehicles are equipped with onboard units (OBUs) to run the proposed routing protocol and a navigation system. Therefore, when sending an Interest message, Content Requesters know their current position and include this information as well as the destination where the content object is requested in the header of the Interest message. Based on this, the NDN Interest message structure is extended by including the Source and Destination fields, to identify the message geographic origin and destination positions, a Time-to-Live (TTL) field, to track the lifetime of Interest messages and a HOPS field, to track the number of hops traveled by the Interest message in addition to the Name and ID fields. Data and Interest message structures differ from each other as Data messages include the Content field while the Source, Destination and Hops fields are not needed since Data messages travel through the same intermediate vehicles that the corresponding Interest messages traveled, according to the NDN Data messages work-flow [1].

Furthermore, we assume the case of at least an average vehicle density, so that Interest messages can propagate towards Content Providers. Investigating the effects of low vehicle densities in VNDN is the scope of our future work.

\section{ROUTING IN VEHICULAR NAMED DATA NETWORKING}

The wireless nature of VANETs has encouraged the development of multi-hop routing schemes able to decrease the number of required message retransmissions and avoid unnecessary usage of network resources. Contention-Based Forwarding (CBF) [8] is a Position-Based Routing (PBR) protocol that relies on distance to select message forwarder nodes in a way that most progress towards the message destination is achieved. The differences between existing PBR approaches lie mainly in the technique used to select the best next hop. Each approach is more suitable for certain scenarios. For instance, in a city scenario area-based approaches [2] perform better whereas in a highway scenario distance-based schemes [8] present lower delays while still providing high ISRs.

PBR schemes can also be divided into two categories according to where the selection of the next hop is done.

In (i) Source-based schemes [9], the sender of a message indicates which neighbor vehicle is more suitable to forward the message. This approach requires that vehicles know the topology of their one-hop network. A common method to acquire this knowledge is through the exchange of beacon messages. However, while the mobility and density of vehicles increase, the number of beacon exchanges also quickly increases, potentially leading to congestion on the communication channel.

In (ii) Receiver-based schemes, each vehicle decides whether to forward a received message, through the use of timers set in a way that vehicles located in better positions are favored. Since Receiverbased schemes are Beacon-less [10], they generate less load on the communication channel compared to Source-based schemes. Moreover, in the case of VNDN, which already present Interest and Data messages, adding extra messages shall be avoided for scalability purposes.

Considering the above stated, our VNDN routing protocol presents the following characteristics: (i) Multi-hop; (ii) Receiver-based; (iii) Beacon-less; and (iv) Distance-based.

Considering the scenario shown in Figure 1, vehicle A sends an Interest message requesting a content object previously advertised by vehicle $J$. The Interest message is first received by the vehicles in the one-hop neighborhood of A (limited by the green ellipse) and one of the following two cases can happen:

(i) One or more of the intermediate vehicles B, C, D, E or F already have a copy of the requested content object. In this case, the corresponding Data message is sent towards A by the vehicle closer to A. 


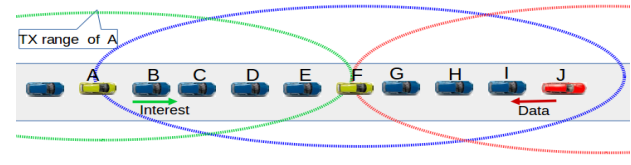

Figure 1: VNDN Routing

(ii) None of the intermediate vehicles have a copy of the requested content object. In such a case the Interest message is forwarded towards $\mathrm{J}$ by the vehicle farther away from A (i.e. $\mathrm{F}$ in this example).

In the first case, to avoid that multiple vehicles answer with the Data message, vehicles schedule the forwarding of the Data message according to a Data timer delay (Data timer delay values are lower than Interest timer delay values). Vehicles closer to the last Interest message forwarder have lower Data timer delay values as shown in Algorithm 1. For example, if only vehicles $\mathrm{C}$ and $\mathrm{E}$ have the requested content object, they will schedule the forwarding of the Data message while vehicles B, D, and F will schedule the forwarding of the Interest message. The timer in $\mathrm{C}$ will expire first and $C$ will send the Data message. When receiving the Data message $\mathrm{E}$ will cancel its scheduled Data message forwarding while $\mathrm{B}, \mathrm{D}$, and $\mathrm{F}$ will cancel their scheduled Interest message forwarding.

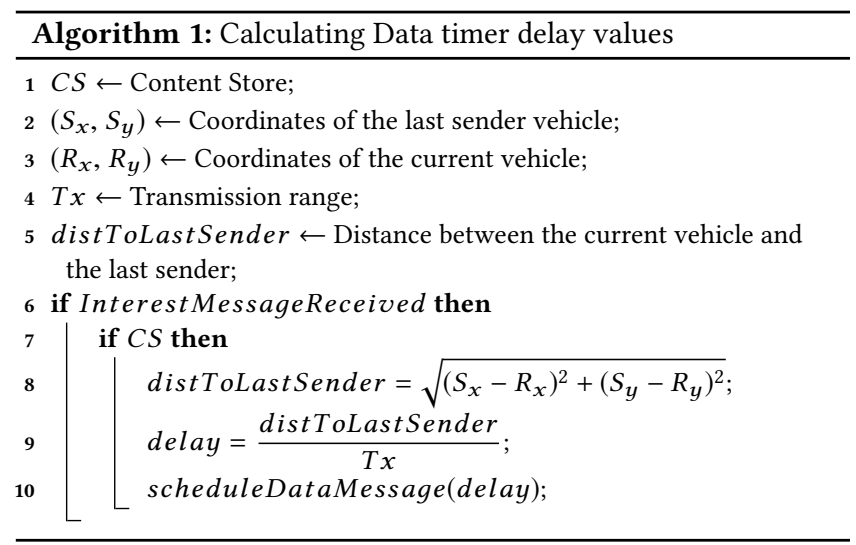

In the second case, all intermediate vehicles schedule the forwarding of the Interest message according to an Interest timer delay. As shown in Algorithm 2, vehicles located farther away from the last sender and that will stay on the road for equal or longer time periods than the maximum expected message delay (i.e. $4 \mathrm{~s}$ for NDN) present shorter Interest timer delay values. Vehicles can obtain their expected remaining time on the road from their navigation system.

The main motivation for choosing as Interest message forwarders, vehicles that will not exit the road in the subsequent four seconds, is to avoid that Interest message forwarder vehicles leave the road before forwarding the corresponding Data messages.

When the Interest timer of a vehicle expires, that vehicle forwards the Interest message and updates its PIT by adding an entry related to the requested content object and consequently becomes a Data Forwarder for that specific content object. The remaining intermediate vehicles, when perceiving that another vehicle has forwarded the Interest message (i.e. the vehicle with shorter timer duration), will cancel their own scheduled Interest message forwarding. In the example shown in Figure 1 the Interest message would be forwarded by vehicle F. The same process repeats until a Content Provider has been reached. The Content Provider then sends the corresponding Data message towards the Content Requester. When intermediate nodes receive the Data message, they perform a PIT lookup and if an entry related to that content object is found, the Data message is forwarded and the PIT entry is removed. Otherwise the Data message is discarded. This process continues until the Data message reaches the original requester or the message is forwarded by the last Data Forwarder vehicle.

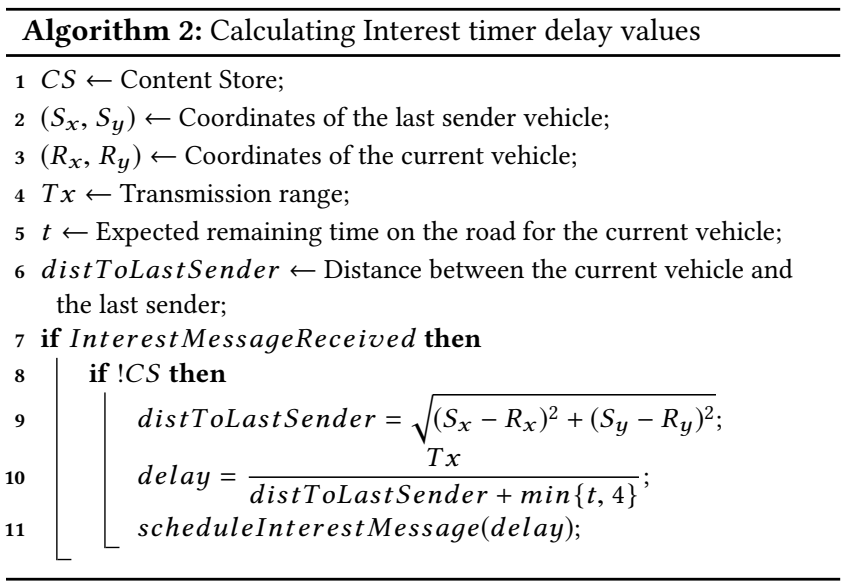

By employing this approach the number of vehicles that forward Interest and Data messages decreases considerably, thus mitigating broadcast storms. To mitigate message redundancy, similarly to [11], intermediate vehicles increase the message HOPS field by one before forwarding Interest messages. With this information whenever a node receives multiple copies of the same Interest message with the same HOPS value, the last copies are recognized as redundant and discarded, thus avoiding incorrect stop of message propagation. On the other hand, if the HOPS value is larger than the value found in a previously received message with the same name and ID, the message is recognized as forwarded by a current one-hop neighbor vehicle and the scheduled message forwarding is canceled. However, as in NDN Data messages travel via the same nodes that forwarded the corresponding Interest message, the RPP problem often happens.

\section{REVERSE PATH PARTITIONING}

In this section we describe the Reverse Path Partitioning (RPP) problem as well as Auxiliary Forwarding Set (AFS), the proposed solution to address RPP.

\subsection{Reverse Path Partitioning Overview}

We define Reverse Path Partitioning (RPP) as disruptions on communication links that prevent Data Forwarders from delivering Data messages to Content Requesters. In cases of high mobility, the probability of RPP increases as the distance between vehicles forwarding Interest messages increases. For better understanding let us consider the following situations: 


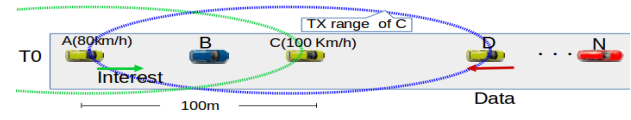

Figure 2: Reverse Path Partitioning

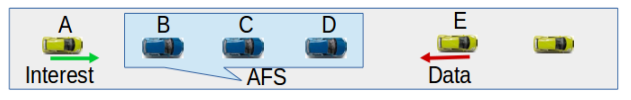

Figure 3: Auxiliary Forwarding Set (i) As illustrated in the example in Figure 2, at time T0 when vehicle $A$ sends an Interest message, the distance between $A$ and $C$ (the next forwarder) is equal to $100 \mathrm{~m}$. However, $A$ is traveling at 80 $\mathrm{km} / \mathrm{h}$ while $C$ is traveling at $100 \mathrm{~km} / \mathrm{h}$. Due to this, later on when the corresponding Data message reaches $\mathrm{C}$, the distance between $\mathrm{C}$ and $\mathrm{A}$ may have increased and A may have moved out of the transmission range of $\mathrm{C}$. Therefore, we say that a partition has occurred on the reverse path since $C$ is not able to deliver the Data message to A, although it has previously forwarded the Interest message received from $A$. This situation can occur between any two consecutive Data Forwarders.

(ii) The transmission range of vehicle A may be larger than for vehicle $C$. This can happen since vehicles may be equipped with devices of different communication capabilities or because antennas in higher vehicles may have better lines of sight and reach larger distances. In this case, $A$ may be able to reach $C$ and thus $C$ can forward an Interest message received from $A$, while $C$ is not able to reach $\mathrm{A}$ and can not deliver the corresponding Data message.

(iii) Signal propagation may suffer temporary attenuation due to the wireless medium conditions and obstacles, which may also prevent $\mathrm{C}$ from delivering the Data message to $\mathrm{A}$.

To mitigate RPP we introduce the concept of Auxiliary Forwarding Set (AFS).

\subsection{Auxiliary Forwarding Set}

The forwarder set idea was proposed in ExOR [9] for a different case. In ExOR, message exchange is always applied between the set of nodes that received a message to agree on the next forwarder. In AFS, only when RPP is detected, the set of vehicles that received a Data message agree on which of them shall also forward the message. Furthermore, message exchange between AFS members is not required since a timer based decision is employed in addition to other input parameters as described below. Unlike in standard NDN, where a single node in each one-hop neighborhood forwards a Data message, vehicles in an AFS are eligible to also forward the Data message in case of RPP.

Let us consider the case shown in Figure 3, where vehicle A forwards an Interest message, which is then forwarded by vehicle E. When vehicles B, C, and D perceive that the Interest message has been forwarded by E, they evaluate the probability of RPP between A and E. If high RPP occurrence probability is detected, they add a PIT entry for the corresponding Data message and form an AFS. To determine the probability of RPP, vehicles take as inputs the road speed limits, the average road speeds, the maximum expected Interest Satisfaction Delays, the transmission range of vehicles and distances between consecutive Interest Forwarders. While road speed limits can be obtained directly from navigation systems, average road speeds can be obtained from navigation services such as WAZE (www.waze.com) or from neighbor Road Side Units (RSUs) that possess real time traffic information. For instance, in Figure
3 for a maximum expected Interest Satisfaction Delay of $4 \mathrm{~s}$ and a transmission range of $200 \mathrm{~m}$, if vehicle $\mathrm{E}$ is traveling at the road speed limit of $100 \mathrm{~km} / \mathrm{h}$ while $\mathrm{A}$ is traveling at the road average speed of $80 \mathrm{~km} / \mathrm{h}$, after $4 \mathrm{~s}$ the distance between $\mathrm{A}$ and $\mathrm{E}$ increases by $22.4 \mathrm{~m}$. Therefore, RPP occurs with high probability if at the time of sending the Interest message the distance between $\mathrm{A}$ and $\mathrm{E}$ is around or larger than $177.6 \mathrm{~m}$ (i.e. transmission range $-22.4 \mathrm{~m}$ ). As explained above, RPP may also happen due to other factors. Due to this, smaller distances shall be considered as of high RPP probability.

When $\mathrm{E}$ forwards the Data message if A does not receive it, the vehicle in the AFS that is farther away from $\mathrm{E}$ (i.e. B in this example) is selected, through a timer based mechanism, to forward the Data message towards A. Vehicles belonging to an AFS use Data messages forwarded by neighbor vehicles as implicit acknowledgments of content reception.

With AFS we address the RPP problem and maintain high Interest Satisfaction Rates regardless of vehicles mobility, which is our main goal.

\section{PERFORMANCE EVALUATION}

\section{Table 1: Simulation parameters}

\begin{tabular}{ll}
\hline Parameters & Values \\
\hline Number of vehicles & $100,200,300,400$ vehicles \\
Vehicles speeds & $50,80,100 \mathrm{Km} / \mathrm{h}$ \\
Number of Content Requesters & $10,20,30,40$ \\
Carrier frequency & $5.9 \mathrm{GHz}$ \\
WAVE channel type & Service Channel \\
Transmission rate & $1 \mathrm{Mbit} / \mathrm{s}$ \\
Size of Interest messages & $1024 \mathrm{bytes}$ \\
Size of data Messages & $4096 \mathrm{bytes}$ \\
Number of chunks per content & 100 \\
Communication technology & IEEE802.11p \\
Communication types & V2V and V2I \\
Maximum transmission range & $200 \mathrm{~m}$ \\
TTL of interest messages & $4 \mathrm{~s}$ \\
Shareability & shareable data \\
\hline
\end{tabular}

For evaluation we deployed the NDN core components such as Interest and Data messages, CS and PIT as well as our VNDN routing scheme in the Omnet++ network simulator [12], we used SUMO [13] for road traffic simulation and VEINS [14] for intervehicular communications.

\subsection{Simulation Parameters}

We simulated the cases of 100, 200, 300 and 400 vehicles driving with maximum speeds of $50 \mathrm{~km} / \mathrm{h}, 80 \mathrm{~km} / \mathrm{h}$ and $100 \mathrm{~km} / \mathrm{h}$, equipped with 802.11p (WAVE) communication capabilities [15]. Table 1 lists the main simulation parameters. 
Since our goal is to understand the effects of receiver mobility apart from other factors, the original Content Provider is static. With this in mind, we used an RSU as original Content Provider, configured with the same parameters compared to the vehicles. Whenever the RSU receives an Interest message, it replies with the corresponding Data message.

\subsection{Metrics and Scenarios}

We use the three following performance metrics:

(i) Interest Satisfaction Rate: Average percentage of Data messages received in response to Interest messages sent;

(ii) Delay per Interest Satisfied: Average time difference between sending an Interest message and receiving the correspondent Data message by a Content Requester;

(iii) Data Forwarded: Average number of Data messages forwarded to neighbors per content object requested;

We used the following two different mobility scenarios:

(i) Scenario I: This scenario is shown in Figure 4 and consists of a $3 \mathrm{~km}$, two lane, one-way road. For this case the original Content Provider (RSU) is placed at a distance $2 \mathrm{~km}$ away from the vehicles starting position along the road;

(ii) Scenario II: This scenario is shown in Figure 5 and consists of a real world 2-way, 4 lanes, $10 \mathrm{Km}$ road with several street junctions along the E45 Route in the city of Erlangen, Germany. For this case the Original Content Provider (RSU) was placed at a roundabout located close to the center of the road at a latitude and longitude of 49.56 and 10.99 degrees respectively.

\subsection{Results}

The results were calculated from the average of 33 simulation runs with a confidence interval of $95 \%$. As a benchmark, first we evaluate the simplistic case of no mobility $(\mathrm{v}=0 \mathrm{~km} / \mathrm{h})$ in scenario I. For this case, vehicles were placed evenly along the road with an intervehicle distance of $100 \mathrm{~m}, 1$ vehicle was selected to request a content object and we obtained an Interest Satisfaction Rate (ISR) of $100 \%$, a delay lower than $1.3 \mathrm{~s}$ and 9 Data messages forwarded. Considering the cases with mobility, as it can be observed here as well as in [5] and [7], when AFS is not applied, the VNDN performance degrades with increasing vehicle speeds. The average ISR decreases and the average delay increases due to the effects of RPP.

When applying AFS the RPP problem is addressed and high ISRs are achieved regardless of neither receiver mobility nor the number of vehicles, at the cost of slightly increasing the amount of space used in PITs, the number of Data messages forwarded and the average delay. Furthermore, the results show that AFS even favor higher speed cases presenting slightly better ISRs for higher speeds. This happens because when vehicle speeds increase compared to average road speeds, more AFSs are formed which better mitigates RPP. However, this also impacts the number of Data messages forwarded as it can be observed in Figures 6, 7 and 8.

Considering the second scenario, the results shown in Figures 9, 10 , and 11 are similar to the obtained in the first scenario, except for a small increase in delays and Data forwarded, which were expected due to the larger distance.
We also assess the AFS scalability using scenario II. We increase the number of content requests from 10 to 40 , with a vehicle speed limit of $100 \mathrm{~km} / \mathrm{h}$. Figure 12 shows that high ISRs, over $96 \%$, are maintained despite a slight decrease when the number of content requests increases. Considering this, we conclude that the proposed solution is not affected by the increase of the number of content requests, which proves its scalability.

These results show that AFS is an efficient and scalable solution to mitigate the effects of receiver mobility under realistic mobility scenarios and enable high VNDN application performance.

\section{CONCLUSIONS}

In this work we investigated VNDNs. We focused on the effects of receiver mobility and proposed a new routing protocol. In particular, we identified the problem of RPP that degrades VNDN application performance when receiver mobility increases and we proposed AFS as a solution. We conducted a series of simulations and showed that our solution is effective and scalable since it is able to provide high VNDN application performance without excessive load on the communication channel regardless of receivers mobility and the number of content requests.

\section{ACKNOWLEDGMENTS}

This work was undertaken under the CONTACT project, CORE/ SWISS/15/IS/10487418, funded by the National Research Fund Luxembourg (FNR) and the Swiss National Science Foundation (SNSF) project no. 164205 . The authors also would like to thank CAPES and FAPESP for the support.

\section{REFERENCES}

[1] Van Jacobson, Diana K Smetters, James D Thornton, Michael F Plass, Nicholas H Briggs, and Rebecca L Braynard. Networking named content. In Proceedings of the 5th international conference on Emerging networking experiments and technologies, pages 1-12. ACM, 2010.

[2] Leandro Aparecido Villas, Azzedine Boukerche, Guilherme Maia, Richard Werner Pazzi, and Antonio AF Loureiro. Drive: An efficient and robust data dissemination protocol for highway and urban vehicular ad hoc networks. Computer Networks, 75:381-394, 2014.

[3] Carlos Anastasiades, Tobias Schmid, Jürg Weber, and Torsten Braun. Informationcentric content retrieval for delay-tolerant networks. Computer Networks, 107: 194-207, 2016

[4] Joao M Duarte, Eirini Kalogeiton, Ridha Soua, Gaetano Manzo, Maria Rita Palattella, Antonio Di Maio, Torsten Braun, Thomas Engel, Leandro A Villas, and Gianluca A Rizzo. A multi-pronged approach to adaptive and context aware content dissemination in vanets. Mobile Networks and Applications, pages 1-13, 2017.

[5] Marica Amadeo, Claudia Campolo, and Antonella Molinaro. Enhancing contentcentric networking for vehicular environments. Computer Networks, 57(16): 3222-3234, 2013.

[6] Giulio Grassi, Davide Pesavento, Giovanni Pau, Rama Vuyyuru, Ryuji Wakikawa, and Lixia Zhang. Vanet via named data networking. In Computer Communications Workshops (INFOCOM WKSHPS), 2014 IEEE Conference on, pages 410-415. IEEE, 2014.

[7] Syed Hassan Ahmed, Safdar Hussain Bouk, Muhammad Azfar Yaqub, Dongkyun Kim, Houbing Song, and Jaime Lloret. Codie: Controlled data and interest evaluation in vehicular named data networks. IEEE Transactions on Vehicular Technology, 65(6):3954-3963, 2016.

[8] Holger Füßler, Hannes Hartenstein, Martin Mauve, Wolfgang Effelsberg, and Jörg Widmer. Contention-based forwarding for street scenarios. In WIT 2004, number LCA-CONF-2004-005, 2004.

[9] Sanjit Biswas and Robert Morris. Exor: opportunistic multi-hop routing for wireless networks. ACM SIGCOMM Computer Communication Review, 35(4): 133-144, 2005

[10] Marc Heissenbüttel, Torsten Braun, Thomas Bernoulli, and Markus Wälchli. Blr: beacon-less routing algorithm for mobile ad hoc networks. Computer communications, 27(11):1076-1086, 2004. 


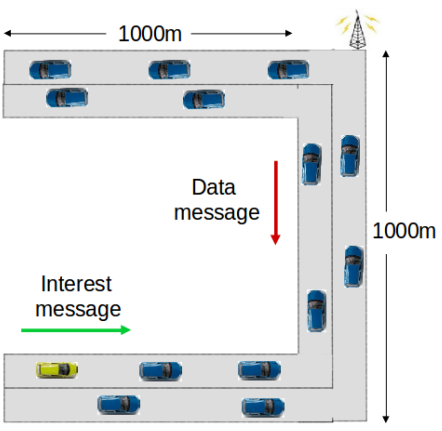

Figure 4: Scenario I

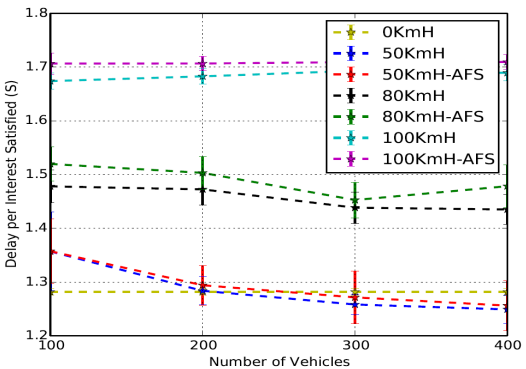

Figure 7: Delay - Scenario I

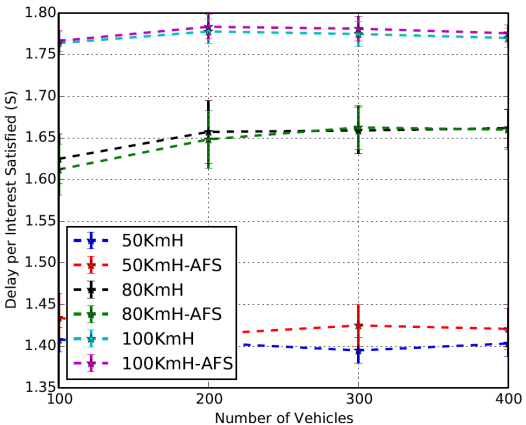

Figure 10: Delay per Interest Satisfied - Scenario II

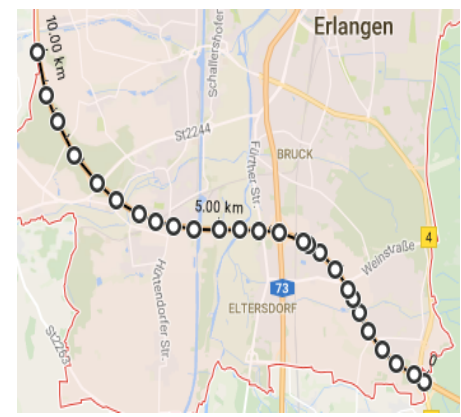

Figure 5: Scenario II

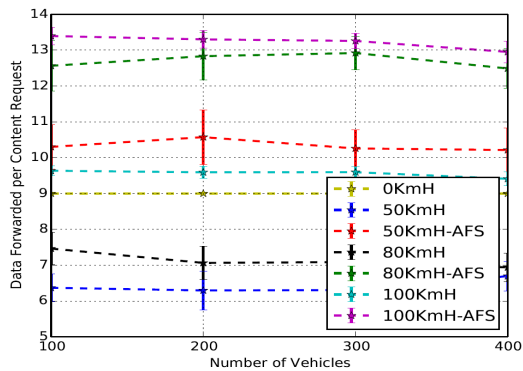

Figure 8: Data Forwarded - Scenario I

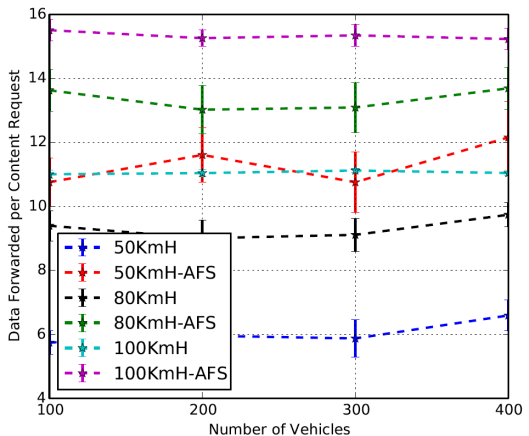

Figure 11: Data messages Forwarded Scenario II

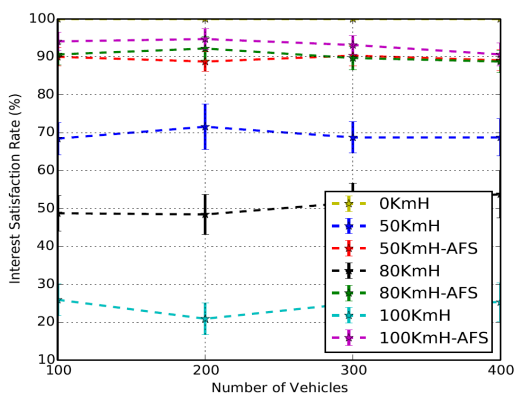

Figure 6: ISR - Scenario I

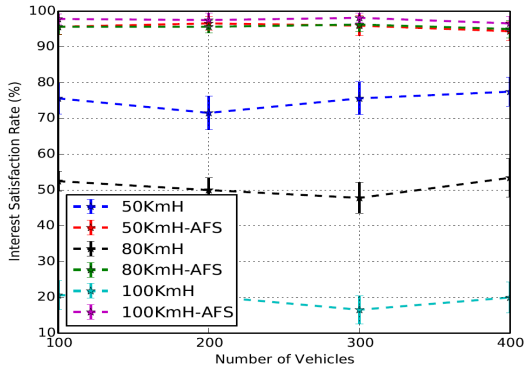

Figure 9: ISR - Scenario II

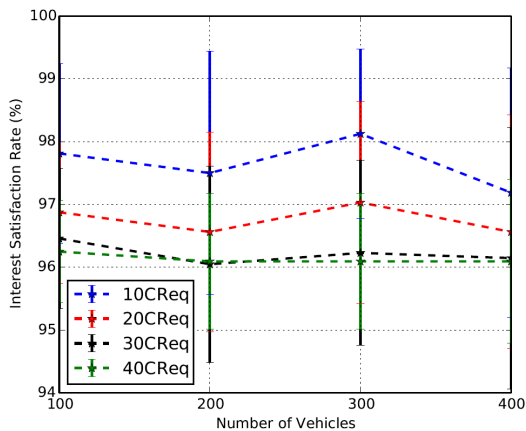

Figure 12: Scalability
[11] Carlos Quadros, Aldri Santos, Mario Gerla, and Eduardo Cerqueira. Qoe-driven dissemination of real-time videos over vehicular networks. Computer Communications, 91:133-147, 2016.

[12] András Varga et al. The omnet++ discrete event simulation system. In Proceedings of the European simulation multiconference (ESM 2001), volume 9, page 65. sn, 2001.

[13] Michael Behrisch, Laura Bieker, Jakob Erdmann, and Daniel Krajzewicz. Sumosimulation of urban mobility: an overview. In Proceedings of SIMUL 2011, The Third International Conference on Advances in System Simulation. ThinkMind, 2011.
[14] Christoph Sommer, Reinhard German, and Falko Dressler. Bidirectionally Coupled Network and Road Traffic Simulation for Improved IVC Analysis. IEEE Transactions on Mobile Computing, 10(1):3-15, January 2011. doi: 10.1109/TMC. 2010.133.

[15] E. Donato, G. Maia, J. M. Duarte, A. A. F. Loureiro, E. Madeira, and L. Villas. Presync: A method for preventing resynchronization in the ieee $802.11 \mathrm{p}$ standard. In ISCC 2015, pages 457-462. IEEE, 2015. 